\title{
Anti-plane interfacial crack with functionally graded coating: static and dynamic
}

\author{
M. Li ${ }^{1}$, Y.L. Tian ${ }^{1}$, P.H. Wen ${ }^{2}$ and M.H. Aliabadi ${ }^{3}$ \\ ${ }^{1}$ College of Mathematics, Taiyuan University of Technology, Taiyuan, China \\ ${ }^{2}$ School of Engineering and Materials Science, Queen Mary, University of London, London E1 4NS, UK \\ ${ }^{3}$ Department of Aeronautics, Imperial College London, South Kensington Campus, London SW7 2BY, UK
}

\begin{abstract}
The anti-plane displacement discontinuity method is applied to establish the Fredholm integral equation of the first kind for the orthotropic Functionally Graded Material (FGM) coatings subjected to static/dynamic shears. The Young's modulus and mass density are assumed to vary exponentially through the thickness while the Poisson's ratio is constant. The static and dynamic fundamental solutions with anti-plane displacement discontinuity are derived for orthotropic FGM coating by using Fourier transform method and Laplace transform method. It has been shown that the transformed fundamental solution with orthotropic coatings has the same order of hyper-singularity as in the static case, i.e. $O\left(1 / r^{2}\right)$, and the Chebyshev polynomials of the second kind are used to solve the integral equations numerically. The time dependent stress intensity factors are obtained directly from the coefficients of the Chebyshev polynomials with the aid of Durbin's Laplace transform inversion method. A comparative study of FGM versus homogeneous coating is conducted, and the dependence of the stress intensity factors in the coating/substrate system on the material property (orthotropic) and thickness of coating is examined. Two examples including the static/dynamic loads are given as benchmarks for the numerical methods and application in composite engineering.
\end{abstract}

Key words: Anti-plane interfacial crack, functionally graded material, coat, displacement discontinuity method, Fredholm integral equation.

Corresponding author: liming04@gmail.com (M.Li) and p.h.wen@qmul.ac.uk (P.H.Wen) 


\section{Introduction}

Coating is a covering that is applied to the surface of an object, usually referred to as the substrate. The purpose of applying the coating may be decorative, functional, or both. The coating itself may be an all-over coating, completely covering the substrate, or it may only cover parts of the substrate. Many industrial coating processes involve the application of a thin film of the functional material to a substrate, such as paper, fabric, film, foil, or sheet stock. If the substrate starts and ends the process wound up in a roll, the process may be termed "roll-toroll" or "web-based" coating. In fiber-reinforced composite materials, coating on fibers is widely employed in order to increase the bonding strength between fibers and matrix, and it is more reasonable to regard an interface as an interphase layer with finite thickness.

The problem of dislocation in the three-phase model has also attracted many investigators' attention, since the obtained solution can be used to study crack growth in composites, as well as strengthening and hardening mechanisms in alloyed materials. Functional coatings may be applied to change the surface properties of the substrate, such as adhesion, corrosion resistance, or wear resistance. In other cases, e.g. semiconductor device fabrication (where the substrate is a wafer), the coating adds a completely new property such as a magnetic response or electrical conductivity and forms an essential part of the finished product. In the FGMs, the variation of the material properties can be pre-determined by controlling the spatial distribution of the composition and the volume fraction of their constituents. These materials have been introduced in recent years to benefit from the ideal performance of its constituents, e.g. the high temperature and the corrosion resistance of the ceramics on one side and the large mechanical strength and toughness of the metals on the other side [1,2]. Direct problems of obtaining temperature, stresses and displacements in a structure in inhomogeneous materials, such as fiber-reinforced composites and FGMs have been studied extensively [3-8].

The fracture analysis concerning interfacial zone has become attractive with the application of the functionally gradient materials in engineering including different modes of fracture [913]. For anti-plane mode III fracture problem, Zhang and Liang [14] investigated a central crack in an inhomogeneous rectangular plane. Shear modulus of the plane varying in the power form along one side of rectangle. Afterwards, Zhang [15] studied an isotropic rectangular sheet subjected to an anti-plane shear with an edge crack off the center line of a rectangle. The problem was solved numerically with a Fredholm integral equation of the second kind using the 
Fourier transform and Fourier series. Erdogan et al [16] studied the anti-plane fracture problems of two bonded homogeneous half planes with a non-homogeneous interfacial zone. By assuming that the medium contains three collinear cracks, the stress intensity factors were obtained for some typical crack geometries and material combinations. Jin and Noda [17] showed that the crack tip fields in general non-homogeneous materials are identical to those in homogeneous materials as long as the material properties are continuous and piece-wise continuously differentiable. Jin and Batra [18,19] investigated the interface cracking between ceramic and/or FGM coatings and a substrate under anti-plane shear with four coating models. For elastodynamic anti-plane fracture problems, a crack at an arbitrary angle to the graded interfacial zone in bonded medias was studied under shear impact with shear modulus and mass density varying in the form of power functions by Choi [20]. Li and Weng [21] carried out a dynamic fracture analysis of a functionally graded interlayer between two coaxial dissimilar homogeneous cylinders subjected to impact torque. Two collinear cracks in a bi-FGM structure perpendicular to the material gradient direction were investigated, and the influences of geometrical and physical parameters on the dynamic stress intensity factor were analyzed by $\mathrm{Li}$ et al [22] using Laplace and Fourier integral transforms. By using Volterra-type edge dislocation, Faal and Dehghan [23] presented the stress analysis for a cracked rectangular sheet made of functionally graded material under anti-plane conditions. For anti-plane interfacial crack in two bonded functionally graded piezoelectric materials, Hu et al [24] shown that the order of singularity of the crack tip stress field and electric displacement is unaffected by the discontinuity of the derivative of material coefficients.

In this paper, we derived the fundamental solutions of displacement discontinuity for antiplane fracture problems and applied them to single layered homogeneous/FGM coatings under both static and dynamic loadings. The Fredholm integral equation of the first kind containing the displacement discontinuity is formulated to determine the stress intensity factors. As the fundamental solutions contain a hyper singular term, the Chebyshev polynomial of the second kind is employed to solve the integral equation. The static and dynamic stress intensity factors are determined from the coefficients of Chebyshev polynomials with high accuracy. Two numerical examples are studied to demonstrate the accuracy and efficiency of the proposed formulation for both static and dynamic problems. 


\section{Fundamental solution of displacement discontinuity with coatings}

Due to the coatings are very thin compared with the substrate, we consider a semi-infinite substrate boned with a coating of thickness $h$ at its surface as shown in Figure 1(a) and the coating-substrate system is subjected to anti-plane shear on the interface crack surfaces. For the sake of convenience of analysis, a Cartesian coordinate (xoy) is selected in Figure 1(a) so that the $x$-axis is along the coating-substrate interface and the $y$-axis normal to it. These two axes are also principal axes of the orthotropic coatings respectively. Figures 1(b) and 1(c) show a single layered homogeneous coating and orthotropic functionally graded coating. The shear modulus are assumed to vary continuously from $\mu_{B}$ at the interface $(y=0)$ to $\mu_{L}$ at the surface of the coating $(y=h)$, and

$$
\mu_{y}=\mu_{B} e^{\beta y / h}, \mu_{x}=\eta^{2} \mu_{y}\left(\eta=\sqrt{\mu_{x} / \mu_{y}}\right)
$$

along $y$-axis and $x$-axis respectively, where non-dimensional constant

$$
\beta=\ln \frac{\mu_{L}}{\mu_{B}}
$$

describes the gradient of the shear modulus which is related to the volume fractions of the constituents of the coating [19], the parameter $\eta$ is constant. For homogeneous coating, $\mu_{B}=\mu_{L}$, and for FGM coating, $\mu_{B}=\mu_{0}$. The shear modulus of the substrate is $\mu_{0}$.

\subsection{Static Green's function with FGM coating}

In general, the equilibrium equation, for anti-plane problem, is

$$
\frac{\partial \tau_{x}}{\partial x}+\frac{\partial \tau_{y}}{\partial y}=0
$$

where $\tau_{x}, \tau_{y}$ are shear stresses, and

$$
\tau_{x}=\mu_{x} \frac{\partial w}{\partial x}, \tau_{y}=\mu_{y} \frac{\partial w}{\partial y},
$$

where $w$ is the anti-plane displacement. Therefore, the governing equation of substrate becomes

$$
\nabla^{2} w^{-}=0
$$

and

$$
\tau_{x}^{-}=\mu_{0} \frac{\partial w^{-}}{\partial x}, \tau_{y}^{-}=\mu_{0} \frac{\partial w^{-}}{\partial y} .
$$




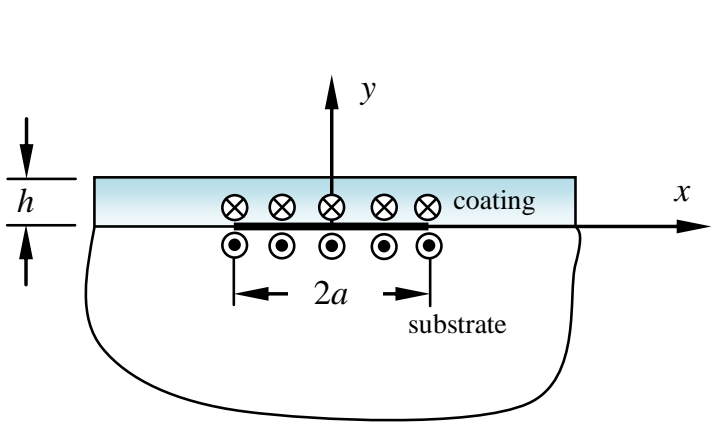

(a)

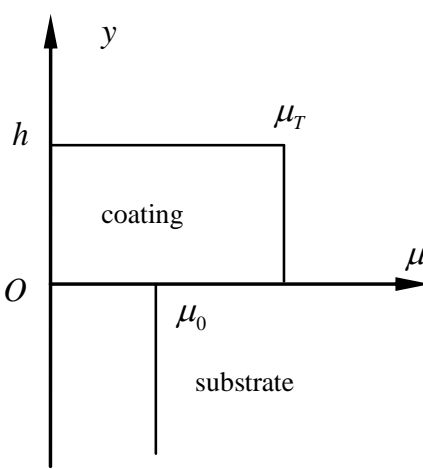

(b)

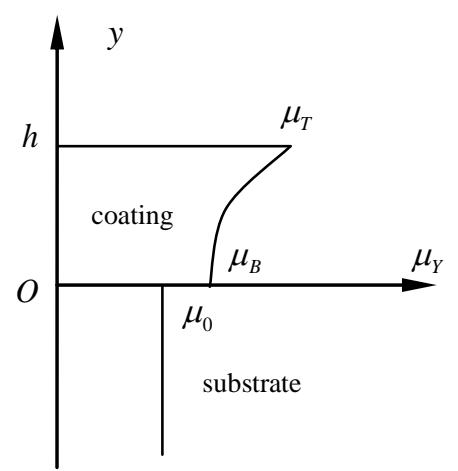

(c)

Figure 1. Single layered coating with an interface crack and variation of shear modulus through the thickness: (a) geometry of interface crack; (b) homogeneous coat; (c) FGM coat.

For the single layered orthotropic FGM model

$$
\begin{aligned}
& \eta^{2} \frac{\partial^{2} w^{+}}{\partial x^{2}}+\frac{\partial^{2} w^{+}}{\partial y^{2}}+\frac{\beta}{h} \frac{\partial w^{+}}{\partial y}=0 \\
& \tau_{x}^{+}=\mu_{B} \eta^{2} e^{\beta y / h} \frac{\partial w^{+}}{\partial x}, \tau_{y}^{+}=\mu_{B} e^{\beta y / h} \frac{\partial w^{+}}{\partial y} .
\end{aligned}
$$

Due to the symmetry about $y$-axis, the general solutions are given

$$
w^{-}=\frac{2}{\pi} \int_{0}^{\infty} C e^{\omega y} \cos \omega x d \omega, y \leq 0
$$

for substrate and

$$
w^{+}=\frac{2}{\pi} \int_{0}^{\infty}\left(A e^{\alpha_{1} y}+B e^{\alpha_{2} y}\right) \cos \omega x d \omega, 0 \leq y \leq h,
$$

for orthotropic coating, where

$$
\alpha_{1}=\sqrt{\eta^{2} \omega^{2}+\frac{\beta^{2}}{4 h^{2}}}-\frac{\beta}{2 h}, \alpha_{2}=-\sqrt{\eta^{2} \omega^{2}+\frac{\beta^{2}}{4 h^{2}}}-\frac{\beta}{2 h},
$$

in which $A, B$ and $C$ are coefficients. For a displacement discontinuity at interface and traction free boundary on the surface of coating, the boundary conditions are described as

$$
\begin{aligned}
& w^{+}-w^{-}=\delta(x), \quad \tau_{y}^{+}-\tau_{y}^{-}=0 \quad y=0 \\
& \tau_{y}^{+}=0 \quad y=h .
\end{aligned}
$$


Thus, we have all coefficients

$$
\begin{aligned}
& C=\frac{1}{\varphi}, \quad A=\frac{\omega E}{\alpha_{1} \kappa \varphi(\varepsilon-1)}, B=-\frac{\omega}{\alpha_{2} \kappa \varphi} \frac{1}{\varepsilon-1} \\
& \varphi=1+\frac{\omega}{\alpha_{2} \kappa(\varepsilon-1)}-\frac{\omega \varepsilon}{\alpha_{1} \kappa(\varepsilon-1)} \\
& \kappa=\frac{\mu_{B}}{\mu_{0}}, \quad \varepsilon=\exp \left(-2 h \sqrt{\eta^{2} \omega^{2}+\beta^{2} / 4 h^{2}}\right) .
\end{aligned}
$$

Then the displacement and shear stress in substrate are written as

$$
\begin{aligned}
& w^{-}(x, y)=\frac{2}{\pi} \int_{0}^{\infty} \frac{1}{\varphi} e^{\omega y} \cos \omega x d \omega, y \leq 0 \\
& \tau_{y}^{-}(x, y)=\frac{2 \mu_{0}}{\pi} \int_{0}^{\infty} \frac{\omega}{\varphi} e^{\omega y} \cos \omega x d \omega, y \leq 0 .
\end{aligned}
$$

It is important to notice that for large value of $\omega$ we have

$$
\frac{\omega}{\varphi} \approx \frac{\kappa \omega \alpha_{2}}{\kappa \alpha_{2}-\omega}=\varphi_{0} \omega+\varphi_{1}+\frac{\varphi_{2}}{\omega}+O\left(\frac{1}{\omega^{2}}\right),
$$

where

$$
\varphi_{0}=\frac{\kappa \eta}{1+\kappa \eta}, \varphi_{1}=\frac{\beta}{2 h} \frac{\kappa}{(1+\kappa)^{2}}, \varphi_{2}=-\frac{\kappa}{1+\kappa \eta} \frac{\kappa \eta-1}{(1+\kappa \eta)^{2}} \frac{\beta^{2}}{8 h^{2} \eta} .
$$

Thus, considering Eq.(15), the shear stress in the substrate can be rearranged, same as in-plane elasticity by Wen et al [25], as

$$
\begin{aligned}
\tau_{y}^{-}(x, y)= & \frac{2 \mu_{0}}{\pi} \int_{0}^{\omega_{0}}\left(\frac{\omega}{\varphi}-\varphi_{0} \omega\right) e^{\omega y} \cos \omega x d \omega+\frac{2 \mu_{0}}{\pi} \int_{\omega_{0}}^{\infty}\left(\frac{\omega}{\varphi}-\varphi_{0} \omega\right) e^{\omega y} \cos \omega x d \omega \\
& +\frac{2 \mu_{0}}{\pi} \int_{0}^{\infty} \varphi_{0} \omega e^{\omega y} \cos \omega x d \omega \\
= & \frac{2 \mu_{0}}{\pi} \int_{0}^{\omega_{0}}\left(\frac{\omega}{\varphi}-\varphi_{0} \omega\right) e^{\omega y} \cos \omega x d \omega+\frac{2 \mu_{0}}{\pi} \int_{0}^{\infty} \varphi_{0} \omega e^{\omega y} \cos \omega x d \omega \\
& +\frac{2 \mu_{0}}{\pi} \int_{\omega_{0}}^{\infty}\left(\varphi_{1}+\frac{\varphi_{2}}{\omega}+O\left(\frac{1}{\omega^{2}}\right)\right) e^{\omega y} \cos \omega x d \omega,
\end{aligned}
$$

where $\omega_{0} h$ should be a large number and $y \leq 0$. Consider integrals

$$
\int_{0}^{\infty} e^{\omega y} \cos \omega x d \omega=\frac{y}{x^{2}+y^{2}}, \int_{0}^{\infty} \omega e^{\omega y} \cos \omega x d \omega=\frac{y^{2}-x^{2}}{\left(x^{2}+y^{2}\right)^{2}}, \int_{z}^{\infty} \frac{1}{u} \cos u d u=-\operatorname{Ci}(z)
$$




$$
\int e^{\omega y} \cos \omega x d \omega=\frac{e^{\omega y}}{x^{2}+y^{2}}(y \cos \omega x+x \sin \omega x)
$$

one may obtain the shear stress along the interface which is called as the fundamental solution with

$$
\begin{aligned}
& Y(x)=\tau_{y}^{-}\left(x, 0^{-}\right) \approx-\frac{2 \mu_{0} \varphi_{0}}{\pi x^{2}}-\frac{2 \mu_{0} \varphi_{0}}{\pi} E(x), \\
& E(x)=-\frac{1}{\varphi_{0}} \int_{0}^{\omega_{0}} G(\omega) \cos \omega x d \omega+\frac{\varphi_{2}}{\varphi_{0}} \operatorname{Ci}\left(\omega_{0} x\right), \\
& G(\omega)=\frac{\omega}{\varphi}-\omega \varphi_{0} .
\end{aligned}
$$

To evaluate the cosine integral, following formula are used

$$
\mathrm{Ci}(z)=\gamma+\ln z+\sum_{k=1}^{\infty} \frac{(-1)^{k} z^{2 k}}{(2 k) ! 2 k}
$$

for small value of $z$, where $\gamma(=0.5772156649)$ is the Euler constant, and for large value of $z$

$$
\mathrm{Ci}(z)=\frac{\sin z}{z} P(z)-\frac{\cos z}{z} Q(z)
$$

in which

$$
P(z)=\sum_{k=0}^{\infty} \frac{(-1)^{k}(2 k) !}{z^{2 k}}, Q(z)=\sum_{k=0}^{\infty} \frac{(-1)^{k}(2 k+1) !}{z^{2 k+1}} .
$$

In the region $0 \leq x \leq \infty$, the function $G(\omega)$ is regular and can be written as

$$
G(0)=0, G(\omega) \approx \varphi_{1}+\frac{\varphi_{2}}{\omega}+O\left(\frac{1}{\omega^{2}}\right)
$$

for large value of $\omega$. It is obvious that the integral in Eq.(18) is convergent for any value of $\omega_{0}$. In addition, the fundamental solution of the shear stress $Y(x)$ in Eq.(20) is of the same order of hyper-singularity $O\left(1 / r^{2}\right)$ as that for an infinite plane with isotropic material. However, there is a weak singularity of order $\ln (r)$ in the cosine integral function $\operatorname{Ci}(z)$. Assuming that in the region $\omega_{2 k} \leq \omega \leq \omega_{2(k+1)}, k=1,2, \ldots, K$, the integral function $G(\omega)$ can be interpolated, by using a polynomial of second order, as 


$$
\begin{aligned}
& G(\omega)=\frac{\left(\omega-\omega_{2 k+1}\right)\left(\omega-\omega_{2 k+2}\right)}{\left(\omega_{2 k}-\omega_{2 k+1}\right)\left(\omega_{2 k}-\omega_{2 k+2}\right)} G_{2 k}+\frac{\left(\omega-\omega_{2 k}\right)\left(\omega-\omega_{2 k+2}\right)}{\left(\omega_{2 k+1}-\omega_{2 k}\right)\left(\omega_{2 k+1}-\omega_{2 k+2}\right)} G_{2 k+1} \\
& +\frac{\left(\omega-\omega_{2 k}\right)\left(\omega-\omega_{2 k+1}\right)}{\left(\omega_{2 k+2}-\omega_{2 k}\right)\left(\omega_{2 k+2}-\omega_{2 k+1}\right)} G_{2 k+2}
\end{aligned}
$$

Substituting $G(\omega)$ into Eq.(20) yields

$$
\begin{aligned}
& \int_{0}^{\omega_{0}} G(\omega) \cos \omega x d \omega \approx \sum_{k=0}^{K}\left\{\frac{1}{2 x^{2} \Delta}\left[\left(G_{2 k}-4 G_{2 k+1}+3 G_{2 k+2}\right) \cos \omega_{2 k+2} x+\left(3 G_{2 k}-4 G_{2 k+1}+G_{2 k+2}\right) \cos \omega_{2 k} x\right]\right. \\
& \left.-\frac{1}{x^{3} \Delta^{2}}\left(G_{2 k}-2 G_{2 k+1}+G_{2 k+2}\right)\left(\sin \omega_{2 k+2} x-\sin \omega_{2 k} x\right)-\frac{1}{x}\left(G_{2 k} \sin \omega_{2 k} x+G_{2 k+2} \sin \omega_{2 k+2} x\right)\right\}
\end{aligned}
$$

where $\Delta=\omega_{0} / 2 K, \omega_{k}=k \Delta$. For numerical examples in this paper, these two free parameters are selected as $K=256$ and $\omega_{0} h=20$ to obtain convergent and accurate fundamental solution of shear stress in Eq.(20).

\subsection{Green's function with FGM coating in Laplace transform domain}

To deal with the anti-plane fracture problem under dynamic load, the Laplace transform method is applied. To observe the effect of modulus gradients on the interfacial crack, the same material of coating is considered as static case. The equilibrium equation, for anti-plane problem, is

$$
\frac{\partial \tau_{x}}{\partial x}+\frac{\partial \tau_{y}}{\partial y}=\rho \frac{\partial^{2} w}{\partial t^{2}}
$$

where $\rho$ is the mass density. Therefore, the governing equation for the substrate becomes

$$
\nabla^{2} w^{-}=\frac{1}{c^{2}} \frac{\partial^{2} w^{-}}{\partial t^{2}}
$$

where $c^{2}=\mu_{0} / \rho_{0}$. However, for orthotropic FGM coating

$$
\begin{aligned}
& \eta^{2} \frac{\partial^{2} w^{+}}{\partial x^{2}}+\frac{\partial^{2} w^{+}}{\partial y^{2}}+\frac{\beta}{h} \frac{\partial w^{+}}{\partial y}=\frac{\rho}{\mu_{y}} \frac{\partial^{2} w^{+}}{\partial t^{2}} \\
& \tau_{x}^{+}=\mu_{B} \eta^{2} e^{\beta y / h} \frac{\partial w^{+}}{\partial x}, \tau_{y}^{+}=\mu_{B} e^{\beta y / h} \frac{\partial w^{+}}{\partial y} .
\end{aligned}
$$

Assume that the density of orthotropic coating varies continuously by

$$
\rho=\rho_{B} e^{\beta y / h}
$$


where $\rho_{B}$ indicates the mass density of coat on the interface. The governing equation is

$$
\eta^{2} \frac{\partial^{2} w^{+}}{\partial x^{2}}+\frac{\partial^{2} w^{+}}{\partial y^{2}}+\frac{\beta}{h} \frac{\partial w^{+}}{\partial y}=\frac{1}{c_{L}^{2}} \frac{\partial^{2} w^{+}}{\partial t^{2}}
$$

where $c_{L}^{2}=\mu_{B} / \rho_{B}$. In this paper, it is assumed that $\rho_{L} / \rho_{B}=\mu_{L} / \mu_{B}=\kappa$, therefore $c_{L}=c$. Applying Laplace transformation over two sides of Eq.(32) with zero initial condition yields

$$
\eta^{2} \frac{\partial^{2} \tilde{w}^{+}}{\partial x^{2}}+\frac{\partial^{2} \tilde{w}^{+}}{\partial y^{2}}+\frac{\beta}{h} \frac{\partial \tilde{w}^{+}}{\partial y}-\frac{p^{2}}{c^{2}} \tilde{w}^{+}=0
$$

where $p$ is Laplace transform parameter and

$$
\tilde{w}(x, y, p)=\int_{0}^{\infty} w(x, y, t) e^{-p t} d t
$$

Again, due to the symmetry about $y$-axis, the general solutions in the Laplace transformed domain are

$$
\tilde{w}^{-}=\frac{2}{\pi} \int_{0}^{\infty} C e^{\tilde{\alpha} y} \cos \omega x d \omega, y \leq 0
$$

for isotropic substrate and

$$
\tilde{w}^{+}=\frac{2}{\pi} \int_{0}^{\infty}\left(A e^{\tilde{\alpha}_{1} y}+B e^{\tilde{\alpha}_{2} y}\right) \cos \omega x d \omega, 0 \leq y \leq h,
$$

for orthotropic coating, where

$$
\tilde{\alpha}=\sqrt{\omega^{2}+\frac{p^{2}}{c^{2}}}, \tilde{\alpha}_{1}=\sqrt{\eta^{2} \omega^{2}+\frac{p^{2}}{c^{2}}+\frac{\beta^{2}}{4 h^{2}}}-\frac{\beta}{2 h}, \tilde{\alpha}_{2}=-\sqrt{\eta^{2} \omega^{2}+\frac{p^{2}}{c^{2}}+\frac{\beta^{2}}{4 h^{2}}}-\frac{\beta}{2 h} .
$$

Considering the boundary conditions for a anti-plane displacement discontinuity at origin on the interface

$$
\begin{aligned}
& w^{+}-w^{-}=\delta(x) \delta(t), \tau_{y}^{+}-\tau_{y}^{-}=0 \quad y=0 \\
& \tau_{y}^{+}=0 \quad y=h
\end{aligned},
$$

gives all coefficients in Eqs (35) and (36) with

$$
\begin{aligned}
& C=\frac{1}{\tilde{\varphi}}, A=\frac{\tilde{\alpha}}{\tilde{\alpha}_{1} \kappa \tilde{\varphi}} \frac{\varepsilon}{\varepsilon-1}, B=-\frac{\tilde{\alpha}}{\tilde{\alpha}_{2} \kappa \tilde{\varphi}} \frac{1}{\varepsilon-1} \\
& \tilde{\varphi}=1+\frac{\tilde{\alpha}}{\tilde{\alpha}_{2} \kappa(\varepsilon-1)}-\frac{\tilde{\alpha} \varepsilon}{\tilde{\alpha}_{1} \kappa(\varepsilon-1)}, \varepsilon=\exp \left(-2 h \sqrt{\eta^{2} \omega^{2}+p^{2} / c^{2}+\beta^{2} / 4 h^{2}}\right) .
\end{aligned}
$$

Similar to static case, the displacement and shear stress in the substrate are given by 


$$
\begin{aligned}
& \tilde{w}^{-}(x, y, p)=\frac{2}{\pi} \int_{0}^{\infty} \frac{1}{\tilde{\varphi}} e^{\tilde{\alpha} y} \cos \omega x d \omega, y \leq 0 \\
& \tilde{\tau}_{y}^{-}(x, y, p)=\frac{2 \mu_{0}}{\pi} \int_{0}^{\infty} \frac{\tilde{\alpha}}{\tilde{\varphi}} e^{\tilde{\alpha} y} \cos \omega x d \omega, y \leq 0 .
\end{aligned}
$$

For large value of $\omega$, we have

$$
\frac{\tilde{\alpha}}{\tilde{\varphi}} \approx \frac{\kappa \tilde{\alpha}_{2}}{\kappa \tilde{\alpha}_{2}-\tilde{\alpha}}=\tilde{\varphi}_{0} \omega+\tilde{\varphi}_{1}+\frac{\tilde{\varphi}_{2}}{\omega}+\tilde{O}\left(\frac{1}{\omega^{2}}\right)
$$

where

$$
\tilde{\varphi}_{0}=\varphi_{0}, \tilde{\varphi}_{1}=\varphi_{1}, \tilde{\varphi}_{2}=\varphi_{2}-\frac{\kappa p^{2}}{2(1+\kappa \eta)^{2}}\left(\frac{\eta}{c^{2}}-\frac{1}{\eta c_{L}^{2}}\right) .
$$

Therefore, the shear stress in the substrate can be written, in the Laplace domain, as

$$
\begin{aligned}
& \tilde{\tau}_{y}^{-}(x, y, p)=\frac{2 \mu_{0}}{\pi} \int_{0}^{\omega_{0}}\left(\frac{\tilde{\alpha}}{\tilde{\varphi}}-\varphi_{0} \omega\right) e^{\tilde{\alpha y}} \cos \omega x d \omega+\frac{2 \mu_{0}}{\pi} \int_{0}^{\infty} \varphi_{0} \omega e^{\tilde{\alpha} y} \cos \omega x d \omega \\
& +\frac{2 \mu_{0}}{\pi} \int_{\omega_{0}}^{\infty}\left(\tilde{\varphi}_{1}+\frac{\tilde{\varphi}_{2}}{\omega}+\tilde{O}\left(\frac{1}{\omega^{2}}\right)\right) e^{\tilde{\alpha} y} \cos \omega x d \omega .
\end{aligned}
$$

Then, the fundamental solution of shear stress along the interface is given

$$
\begin{aligned}
& \tilde{Y}(x, p)=\tilde{\tau}_{y}^{-}\left(x, 0^{-}, p\right) \approx-\frac{2 \mu_{0} \varphi_{0}}{\pi x^{2}}-\frac{2 \mu_{0} \varphi_{0}}{\pi} \tilde{E}(x, p), \\
& \tilde{E}(x, p)=-\frac{1}{\varphi_{0}} \int_{0}^{\omega_{0}} \tilde{G}(\omega, p) \cos \omega x d \omega+\frac{\tilde{\varphi}_{2}}{\varphi_{0}} \operatorname{Ci}\left(\omega_{0} x\right), \\
& \tilde{G}(\omega, p)=\frac{\tilde{\alpha}}{\tilde{\varphi}}-\omega \varphi_{0} .
\end{aligned}
$$

In the region $0 \leq \omega \leq \infty$, the function $\tilde{G}(\omega, p)$ is regular and $\tilde{G}(\omega, p) \approx \frac{\tilde{\varphi}_{2}}{\omega}+\tilde{O}\left(\frac{1}{\omega^{2}}\right)$ for large value of $\omega$. therefore, function $\tilde{E}(x, p)$ is convergent for any large values of $\omega_{0}$. Again, the fundamental solution of the shear stress $\tilde{Y}(x, p)$ is of hyper-singularity $\left(1 / r^{2}\right)$ and the cosine integral function $\mathrm{Ci}(z)$ a weak singularity of $\ln (r)$.

\section{Fundamental solution of strip with GFM coating}

\subsection{Static free strip Green's function with FGM coating}


Consider a strip of width $D$ with two-free-edges as shown in Figure 2. For an anti-plane displacement discontinuity on interface, the boundary conditions can be described as

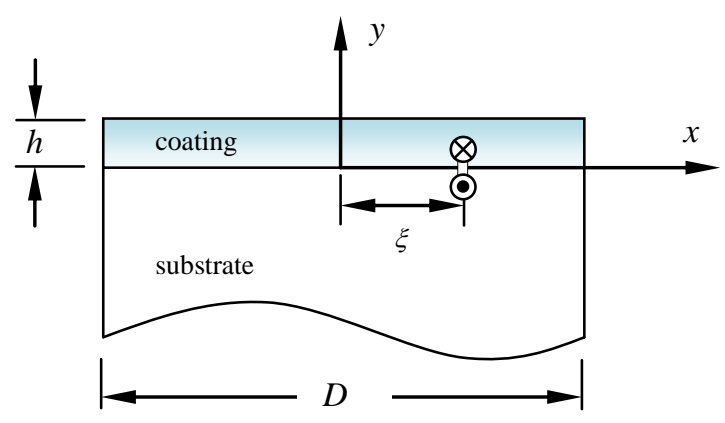

Figure 2. A displacement discontinuity on the interface in a strip.

$$
\begin{aligned}
& w^{+}-w^{-}=\delta(x-\xi), \tau_{y}^{+}-\tau_{y}^{-}=0 \quad y=0, \\
& \tau_{y}^{+}=0 \quad y=h, \\
& \tau_{x}^{+}=\tau_{x}^{-}=0 \quad x= \pm D / 2 .
\end{aligned}
$$

By the principle of superposition, the shear stress in substrate can be arranged as

$$
\begin{aligned}
& \tau_{y}^{-}(\xi, x, y)=\frac{2 \mu_{0}}{\pi} \sum_{k=-\infty}^{\infty} \int_{0}^{\omega_{0}}\left(\frac{\omega}{\varphi}-\varphi_{0} \omega\right) e^{\omega y} F_{k}^{+}(\omega, \xi, x) d \omega+\frac{2 \mu_{0}}{\pi} \sum_{k=-\infty}^{\infty} \int_{0}^{\infty} \varphi_{0} \omega e^{\omega y} F_{k}^{+}(\omega, \xi, x) d \omega \\
& +\frac{2 \mu_{0}}{\pi} \sum_{k=-\infty}^{\infty} \int_{\omega_{0}}^{\infty}\left(\varphi_{1}+\frac{\varphi_{2}}{\omega}+O\left(\frac{1}{\omega^{2}}\right)\right) e^{\omega y} F_{k}^{+}(\omega, \xi, x) d \omega,
\end{aligned}
$$

where

$$
F_{k}^{+}(\omega, \xi, x)=\cos \omega(x-\xi+2 k D)+\cos \omega(x+\xi+2 k D+D) .
$$

One can obtain the fundamental solution of shear stress (Green's function) along the interface as

$$
\begin{aligned}
& Y(\xi, x)=\tau_{y}^{-}(\xi, x, 0)=-\frac{2 \mu_{0} \varphi_{0}}{\pi(x-\xi)^{2}}-\frac{2 \mu_{0} \varphi_{0}}{\pi} E^{+}(\xi, x) \\
& E^{+}(\xi, x)=-\frac{1}{\varphi_{0}} \sum_{k=-\infty}^{\infty} \int_{0}^{\omega_{0}} G(\omega) F_{k}^{+}(\omega, \xi, x) d \omega+\sum_{\substack{k=-\infty \\
k \neq 0}}^{\infty} \frac{1}{(x-\xi+2 k D)^{2}}+\sum_{k=-\infty}^{\infty} \frac{1}{(x+\xi+2 k D+D)^{2}} \\
& +\frac{\varphi_{2}}{\varphi_{0}} \sum_{k=-\infty}^{\infty}\left(\operatorname{Ci}\left[\omega_{0}(x-\xi+2 k D)\right]+\operatorname{Ci}\left[\omega_{0}(x+\xi+2 k D+D)\right]\right),
\end{aligned}
$$


where $G(\omega)$ is defined in Eq.(20). Due to the numerical computational process is convergence, the number of truncation term in the summation in Eq.(50) is taken to 6.

\subsection{Static simply supported strip Green's function with FGM coating}

For a simply supported strip with displacement discontinuity on interface, the boundary conditions are given as

$$
\begin{aligned}
& w^{+}-w^{-}=\delta(x-\xi), \quad \tau_{y}^{+}-\tau_{y}^{-}=0 \quad y=0, \\
& \tau_{y}^{+}=0 \quad y=h, \\
& w^{+}=w^{-}=0 \quad x= \pm D / 2 .
\end{aligned}
$$

By the principle of superposition, the shear stress in substrate is given by

$$
\begin{aligned}
& \tau_{y}^{-}(\xi, x, y)=\frac{2 \mu_{0}}{\pi} \sum_{k=-\infty}^{\infty} \int_{0}^{\omega_{0}}\left(\frac{\omega}{\varphi}-\varphi_{0} \omega\right) e^{\omega y} F_{k}^{-}(\omega, \xi, x) d \omega+\frac{2 \mu_{0}}{\pi} \sum_{k=-\infty}^{\infty} \int_{0}^{\infty} \varphi_{0} \omega e^{\omega y} F_{k}^{-}(\omega, \xi, x) d \omega \\
& +\frac{2 \mu_{0}}{\pi} \sum_{k=-\infty}^{\infty} \int_{\omega_{0}}^{\infty}\left(\varphi_{1}+\frac{\varphi_{2}}{\omega}+O\left(\frac{1}{\omega^{2}}\right)\right) e^{\omega y} F_{k}^{-}(\omega, \xi, x) d \omega,
\end{aligned}
$$

where

$$
F_{k}^{-}(\omega, \xi, x)=\cos \omega(x-\xi+2 k D)-\cos \omega(x+\xi+2 k D+D) .
$$

The Green's function of shear stress along the interface is obtained as

$$
\begin{aligned}
& Y(\xi, x)=\tau_{y}^{-}(\xi, x, 0)=-\frac{2 \mu_{0} \varphi_{0}}{\pi(x-\xi)^{2}}+\frac{2 \mu_{0} \varphi_{0}}{\pi} E^{-}(\xi, x) \\
& E^{-}(\xi, x)=-\frac{1}{\varphi_{0}} \sum_{k=-\infty}^{\infty} \int_{0}^{\omega_{0}} G(\omega) F_{k}^{-}(\omega, x, b) d \omega+\sum_{\substack{k=-\infty \\
k \neq 0}}^{\infty} \frac{1}{(x-\xi+2 k D)^{2}}-\sum_{k=-\infty}^{\infty} \frac{1}{(x+\xi+2 k D+D)^{2}} \\
& +\frac{\varphi_{2}}{\varphi_{0}} \sum_{k=-\infty}^{\infty}\left(\operatorname{Ci}\left[\omega_{0}(x-\xi+2 k D)\right]-\operatorname{Ci}\left[\omega_{0}(x+\xi+2 k D+D)\right]\right) .
\end{aligned}
$$

\subsection{Green's function for strip with FGM coating under dynamic load}

Considering interfacial crack with functionally graded coating under dynamic load, the shear stress in substrate can be arranged, in the Laplace transform domain, as

$$
\tilde{\tau}_{y}^{-}(\xi, x, y, p)=\frac{2 \mu_{0}}{\pi} \sum_{k=-\infty}^{\infty} \int_{0}^{\omega_{0}}\left(\frac{\alpha}{\tilde{\varphi}}-\varphi_{0} \omega\right) e^{\alpha y} F_{k}^{ \pm}(\omega, \xi, x) d \omega+\frac{2 \mu_{0}}{\pi} \sum_{k=-\infty}^{\infty} \int_{0}^{\infty} \varphi_{0} \omega e^{\alpha y} F_{k}^{ \pm}(\omega, \xi, x) d \omega
$$




$$
+\frac{2 \mu_{0}}{\pi} \sum_{k=-\infty}^{\infty} \int_{\omega_{0}}^{\infty}\left(\varphi_{1}+\frac{\tilde{\varphi}_{2}}{\omega}++O\left(\frac{1}{\omega^{2}}\right)\right) e^{\alpha y} F_{k}^{ \pm}(\omega, \xi, x) d \omega
$$

where function $F_{k}^{ \pm}(\omega, \xi, x)$ is given in Eq.(48) for two-free-edge strip and in Eq.(53) for the simply supported strip. Then the fundamental solution of shear stress, in the Laplace transform domain, is

$$
\begin{aligned}
& \tilde{Y}(\xi, x, p)=\tau_{y}^{-}(\xi, x, 0, p)=-\frac{2 \mu_{0} \varphi_{0}}{\pi(x-\xi)^{2}}-\frac{2 \mu_{0} \varphi_{0}}{\pi} \tilde{E}^{ \pm}(\xi, x, p) \\
& \tilde{E}^{ \pm}(\xi, x, p)=-\frac{1}{\varphi_{0}} \sum_{k=-\infty}^{\infty} \int_{0}^{\omega_{0}} G(\omega) F_{k}^{ \pm}(\omega, \xi, x) d \omega+\sum_{\substack{k=-\infty \\
k \neq 0}}^{\infty} \frac{1}{(x-\xi+2 k D)^{2}} \pm \sum_{k=-\infty}^{\infty} \frac{1}{(x+\xi+2 k D+D)^{2}} \\
& +\frac{\tilde{\varphi}_{2}}{\varphi_{0}} \sum_{k=-\infty}^{\infty}\left(\operatorname{Ci}\left[\omega_{0}(x-\xi+2 k D)\right] \pm \operatorname{Ci}\left[\omega_{0}(x+\xi+2 k D+D)\right]\right),
\end{aligned}
$$

for two-free-edge strip (+) and simply supported strip (-) respectively.

It is clear that changing $G(\omega)$ and $\varphi_{2}$ in Eqs (50) and (55) with $\tilde{G}(\omega, p)$ and $\tilde{\varphi}_{2}$ in Eqs (45) and (43) results the dynamic fundamental solutions of displacement discontinuity in the Laplace space at coordinate $(\xi, 0)$.

\section{Integral equation of displace discontinuity for interface crack}

Consider a anti-plane crack and assume the density of the discontinuity displacement $\psi(x)\left(=\Delta w=\left[w^{+}-w^{-}\right]\right)$on the crack surfaces in the region $-a \leq x \leq a$, where $a$ is the half length of crack as shown in Figure 1(a). The integral equation with the density of displacement discontinuity on the crack surface can be written as

$$
\int_{-a}^{a} Y(x-\xi) \psi(\xi) d \xi=-\tau_{0}(x), \quad-a \leq x \leq a
$$

where $\tau_{0}(x)$ is specified shear on the crack surfaces. Take the second degree of Hadamard's finite part from the fundamental solutions in Eqs (20), (49) and (54) for static case, the integral equation (59) can be rewritten as

$$
\int_{-a}^{a} \frac{\psi(\xi)}{|x-\xi|^{2}} d \xi+\int_{-a}^{a} E(x-\xi) \psi(\xi) d \xi=\frac{\pi \tau_{0}(x)}{2 \varphi_{0} \mu_{0}}, \quad-a \leq x \leq a
$$


in which the function $E(x)$ is of weak singular $O(\ln r)$. It is because that the shear stress is of singularity $O\left(r^{-1 / 2}\right)$ at crack tips $x= \pm a$, we assume that

$$
\psi(x)=\sqrt{a^{2}-x^{2}} \sum_{k=0}^{K} c_{k} U_{k}(x / a),
$$

where $c_{k}$ represents the coefficient, $U_{k}(x / a)$ are the Chebyshev polynomials of the second kind, i.e.

$$
U_{k}(x / a)=\frac{\sin [(k+1) \arccos (x / a)]}{\sin [\arccos (x / a)]} .
$$

Due to the integral formula given by Kaya and Erdogan [26],

$$
\int_{-a}^{a} \frac{\sqrt{a^{2}-\xi^{2}} U_{k}(\xi / a)}{\left|x_{i}-\xi\right|^{2}} d \xi=-\pi(k+1) U_{k}\left(x_{i} / a\right)
$$

where the collocation points $x_{i}$ are chosen as

$$
x_{k} / a=\cos \left(\frac{(2 i+1)}{2(K+1)} \pi\right), i=0,1,2, \ldots, K
$$

then Eq.(60) at collocation point $x_{i}$ becomes

$$
\sum_{k=0}^{K} c_{k}\left[-\pi(k+1) U_{k}\left(x_{i} / a\right)+\int_{-a}^{a} E\left(x_{i}-\xi\right) \sqrt{a^{2}-\xi^{2}} U_{k}(\xi / a) d \xi\right]=\frac{\pi \tau_{0}\left(x_{i}\right)}{2 \varphi_{0} \mu_{0}}, i=0,1, \ldots, K
$$

In order to cancel the weak singularity in $E\left(x_{i}-\xi\right)$, the coordinate transformation is applied simply, i.e. $\xi^{\prime}=\sqrt{\xi-x_{i}}$. Eq.(65) provides a set of linear system of equations with $K+1$ unknowns $c_{k}$ to be determined. Finally stress intensity factors can be determined directly by displacement discontinuity from Eq.(61) as follows

$$
K_{I I I}( \pm a)=2 \varphi_{0} \mu_{0} \sum_{k=0}^{K} c_{k} U_{k}( \pm 1) \sqrt{\pi a},
$$

From the properties of the Chebyshev polynomials, $U_{k}(+1)=k+1$ and $U_{k}(-1)=(-1)^{k}(k+1)$. In the following numerical examples, the free parameter $K$ in Eq.(65) is taken to 9.

In the same way, the density of the discontinuity displacement in the transformed domain $\tilde{\psi}(x, p)\left(=\Delta \tilde{w}=\left[\tilde{w}^{+}-\tilde{w}^{-}\right]\right)$on the crack surfaces. The integral equation with the density of displacement discontinuity on the crack surface can be written as 


$$
\int_{-a}^{a} \tilde{Y}(x-\xi, p) \tilde{\psi}(\xi) d \xi=-\tilde{\tau}_{0}(x, p), \quad-a \leq x \leq a
$$

where $\tilde{\tau}_{0}(x, p)$ is transformed shear stress on crack surfaces in the Laplace domain. Considering the fundamental solution in Eq.(45) gives

$$
\int_{-a}^{a} \frac{\tilde{\psi}(\xi, p)}{|x-\xi|^{2}} d \xi+\int_{-a}^{a} \tilde{E}(x-\xi, p) \tilde{\psi}(\xi, p) d \xi=\frac{\pi \tilde{\tau}_{0}(x, p)}{2 \varphi_{0} \mu_{0}}, \quad-a \leq x \leq a
$$

Same as static case, we assume that

$$
\tilde{\psi}(x, p)=\sqrt{a^{2}-x^{2}} \sum_{k=0}^{K} \tilde{c}_{k}(p) U_{k}(x / a)
$$

where $\tilde{c}_{k}$ denotes the coefficient. Then the integral Eq.(68) at collocation point $x_{i}$ becomes

$$
\sum_{k=0}^{K} \tilde{c}_{k}\left[-\pi(k+1) U_{k}\left(x_{i} / a\right)+\int_{-a}^{a} \tilde{E}\left(x_{i}-\xi, p\right) \sqrt{a^{2}-\xi^{2}} U_{k}(\xi / a) d \xi\right]=\frac{\pi \tilde{\tau}_{0}\left(x_{i}, p\right)}{2 \varphi_{0} \mu_{0}}, i=0,1,2, \ldots, K
$$

Finally the stress intensity factors can be determined directly by displacement discontinuity in (40) as follows:

$$
\tilde{K}_{I I I}( \pm a, p)=2 \varphi_{0} \mu_{0} \sum_{k=0}^{K} \tilde{c}_{k}(p) U_{k}( \pm 1) \sqrt{\pi a} .
$$

Selecting $(L+1)$ samples in the transformation space $p_{l}, l=0,1, \ldots, L, \tilde{f}\left(p_{l}\right)$ is evaluated for each Laplace parameter from Eq.(70). Thereafter, function $f(t)$ in the time domain can be determined by the Laplace inversion techniques. A simple and accurate method proposed by Durbin [27] is adopted as follows

$$
f(t)=\frac{2 e^{\sigma / T}}{T}\left[-\frac{1}{2} \tilde{f}\left(p_{0}\right)+\sum_{l=0}^{L} \operatorname{Re}\left\{\tilde{f}\left(p_{l}\right) e^{2 l \pi t i / T}\right\}\right]
$$

where $\tilde{f}\left(p_{l}\right)$ denotes the transformed value of function in the Laplace domain, the parameter of the Laplace transform is chosen as: $p_{l}=(\sigma+2 l \pi i) / T(i=\sqrt{-1})$. In the Durbin's inverse formula Eq.(72) there are two free normalised parameters in $p_{l}: \sigma$ and $T$ in the Durbin's inverse method. In fact, the parameter $T$ depends on the observing period in time domain and $\sigma$ is taken to 5 . 


\section{Numerical examples and discussion}

\section{Example 5.1 Interfacial crack in functionally graded media under static load}

It was mentioned in [19] that in high temperature applications, the coatings are generally made from ceramics and their moduli are usually higher than that of the metal substrate. However, in some cutting tool applications, the modulus of a coating may be lower than that of the substrate. In this observation, a single layered homogeneous coating $\left(\mu_{B}=\mu_{L}\right)$ of the thickness $h$ and single layered orthotropic FGM coatings $\left(\mu_{B}=\mu_{0}\right)$ are considered. To compare the results with different methods, Figure 3 shows the variations of the SIFs with isotropic homogeneous/FGM coatings $(\eta=1)$ versus different parameter $\kappa$ and different ratios $a / h$. The integral equation method was applied in [19] by Jin and Batra for the same problem and the results are found to be the same as that by displacement discontinuity approach in this paper with both single layered homogeneous and isotropic FGM coatings. It can be found that when $\kappa \geq 1$, the SIF with FGM coatig is slightly lower than that of homogeneous coat which indicates that, in terms of SIF reduction, the FGM coat is a better alternative to the conventional homogeneous coat. However, when $\kappa=0.5$, the SIF for FGM coat is slightly larger with $5 \%$ than that of homogeneous coat. But it can be seen that the FGM coatings may be still be superior to homogeneous coatings as they usually provide better bonding strength between the coating and substrate to reduce thermal residual stresses and higher fracture toughness [19].

In general case the composites are anisotropic and the properties of material is directionally dependent. Therefore, it is worth to observe the effect of material property on the SIF. Figures from 4 to 5 show the results of normalized SIF for both two kinds of coating versus the selections of two material parameters $\kappa$ and $\eta$. Again, the material of substrate is isotropic. It is obvious that when both ratios $\kappa$ and $\eta$ increase, the SIFs decrease significantly. For all cases, when the crack length is very small compared with the coating thickness $(a / h<<1)$, the normalised stress intensity factors (SIF) tend to unit, which is the same as a interfacial crack between two semi-infinite dissimilar plane. For FGM coating, when $\kappa \geq 2$, it is evident that while normalized crack length $a / h$ increases from zero to 0.5 , the normalized SIFs decrease with maximum reduction around 5\%. 
A strip with a central crack and traction free boundary condition subjected to uniformly distributed shear on the crack surfaces is observed too. For the sake of convenience of analysis, isotropic FGM coating $(\eta=1)$ is considered with the ratio of $a / h=1$. The normalized SIF variations versus $a / D$ for two different coatings are shown in Figures 6(a) and 6(b) while the parameter $\kappa$ is taken to $0.5,1$ and 2 respectively. In the computational process, the number of truncation in summation Eq.(50) is taken to 5. As expected when $2 a / D$ tends to 1 (when crack tip is closer to the boundary), the normalized SIF increases sharply due to the effect of free traction boundary.

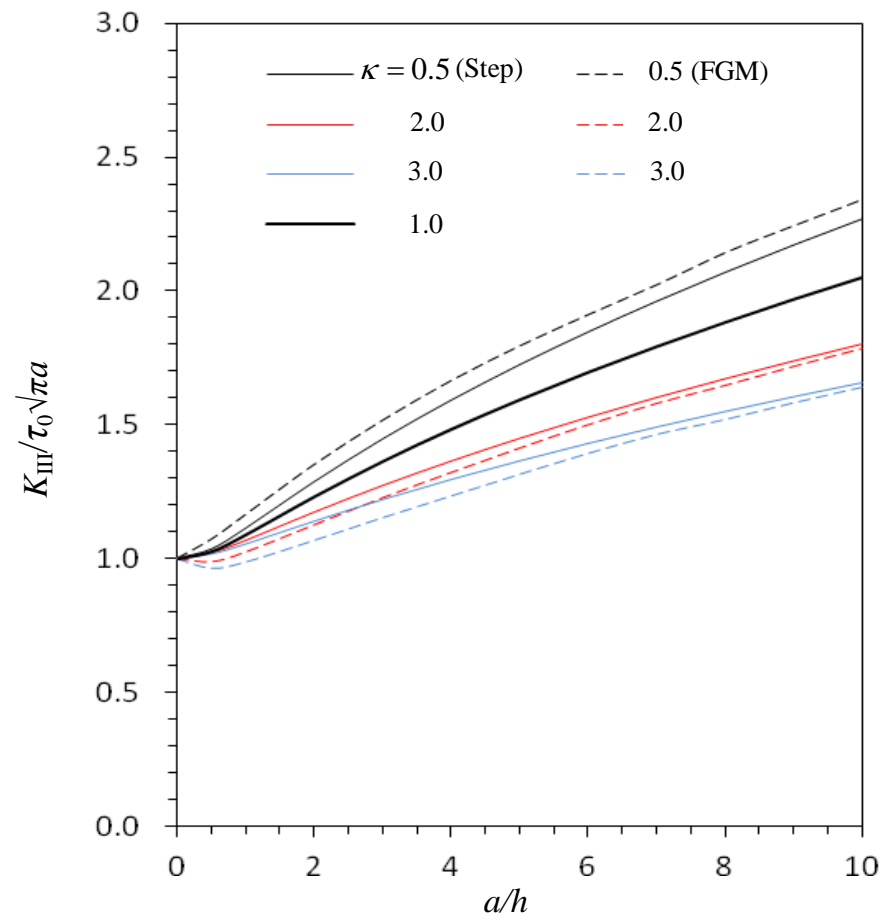

Figure 3. Normalised SIFs for different ratios of $\kappa$ for homogeneous isotropic coating in solid lines and FGM isotropic coating in dashlines. 

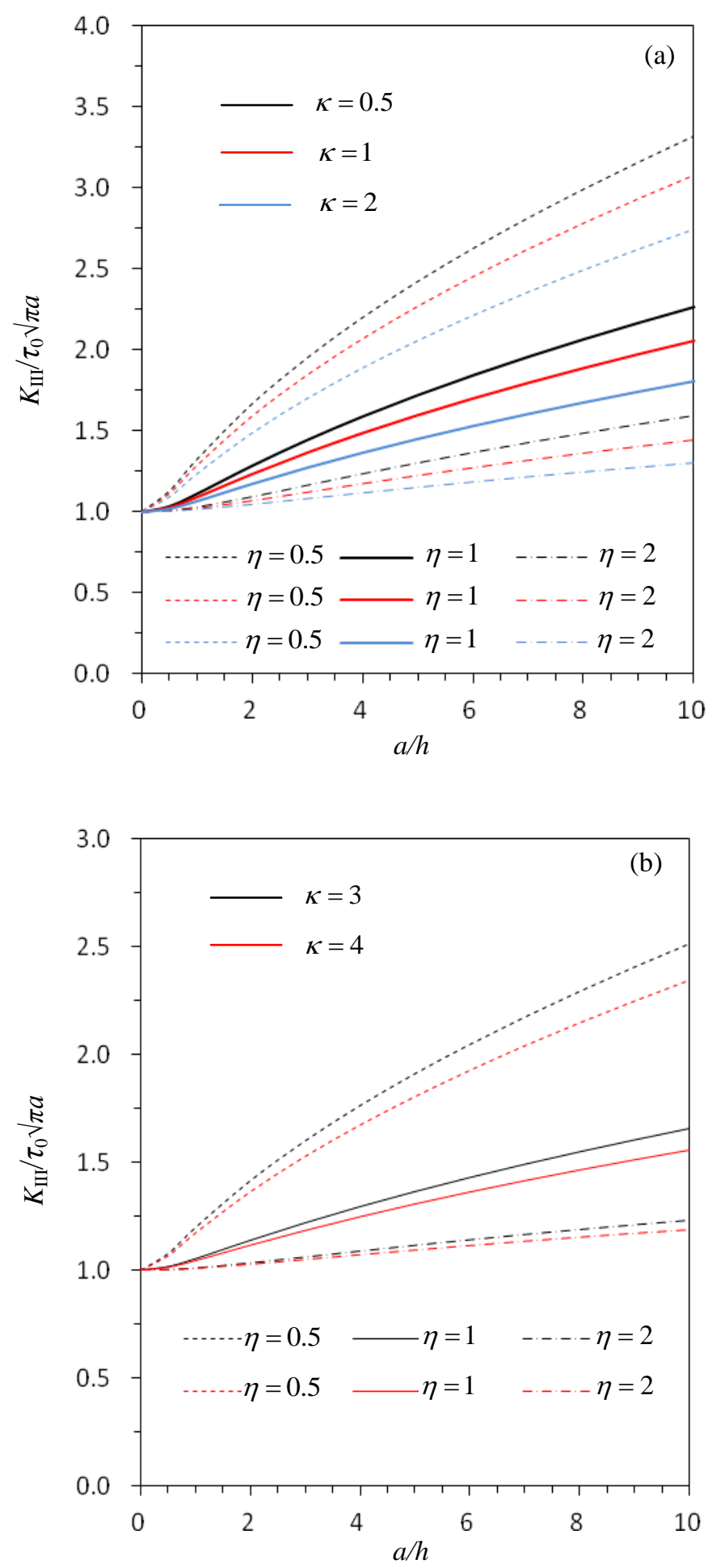

Figure 4. Normalised SIFs for different ratios of $\kappa$ and orthotropic parameters $\eta$ for single layered homogeneous coating. 

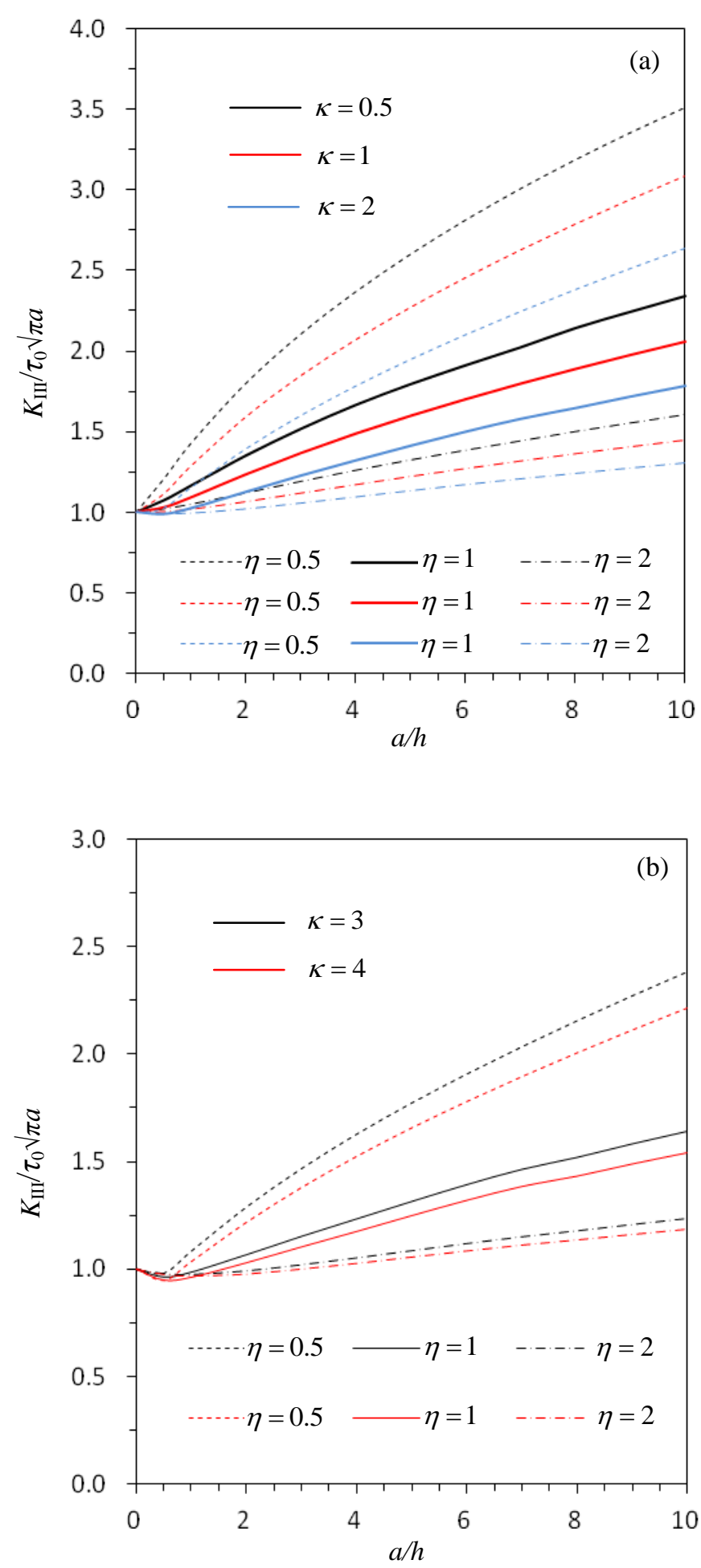

Figure 5. Normalised SIFs for different ratios of $\kappa$ and orthotropic parameters $\eta$ for single layered FGM coating. 

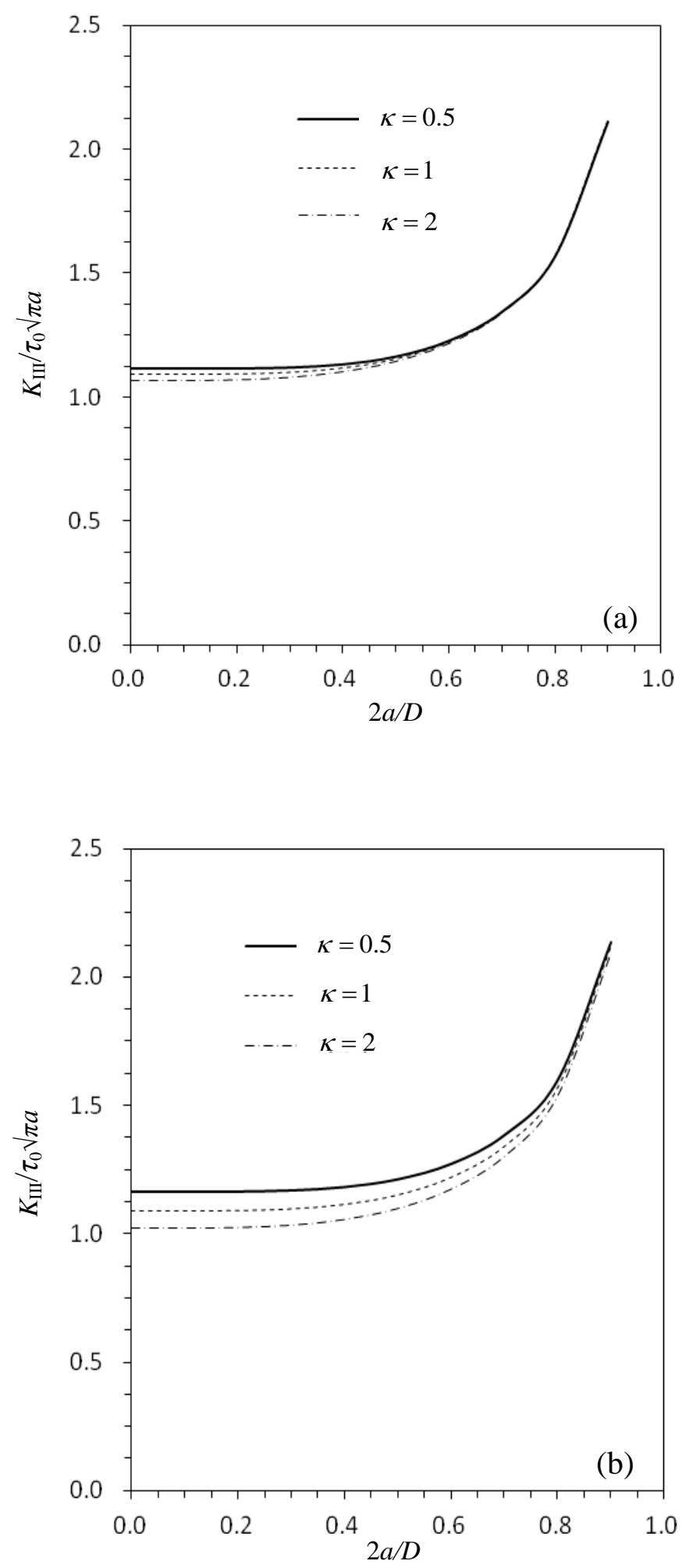

Figure 6. Normalised SIFs with centered crack in the strip for different ratios of $\kappa$ : (a) homogeneous isotropic coating; (b) FGM isotropic coating. 
Example 5.2 Interfacial crack in functionally graded media under dynamic load

Consider a interfacial crack of length $2 a$ between substrate and single layered coating subjected to uniformly distributed shear $\tau_{0} H(t)$ on the crack surfaces, where $H(t)$ is Heaviside function. The number of sample in the Laplace space are chosen as $L=200$ with two free parameters $\sigma=5, T=20$ in the Durbin's inversion method. Two thicknesses of the coating are selected in this example, i.e. $h=a$ and $h=5 a$. In homogeneous/FGM coatings, constant of material $\kappa=2$ and the coefficient $\eta$ is taken to $0.5,1$ and 2 respectively. There is a shear elastic wave with speeds $c\left(=\sqrt{\mu_{0} / \rho_{0}}\right)$ in substrate, and $c_{y}(=c), c_{x}(=\eta c)$ in coat along $y$-axis and $x$-axis respectively. The normalized stress intensity factors $K_{I I I}(t)$ to $\tau_{0} \sqrt{\pi a}$ for two different height $(h=5 a, h=a)$ versus the normalised time $c t / a$ are shown in Figures 7(a) and 7(b) for both homogeneous and FGM coatings respectively.

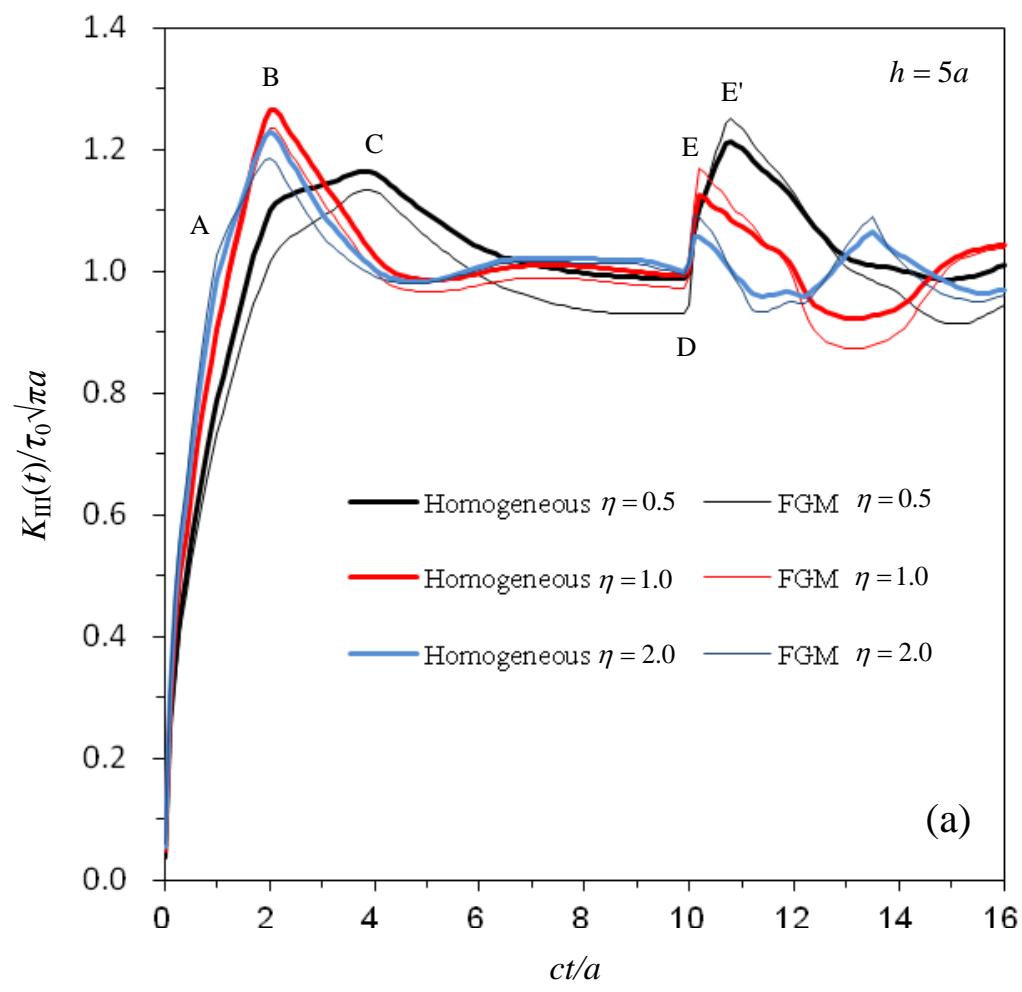




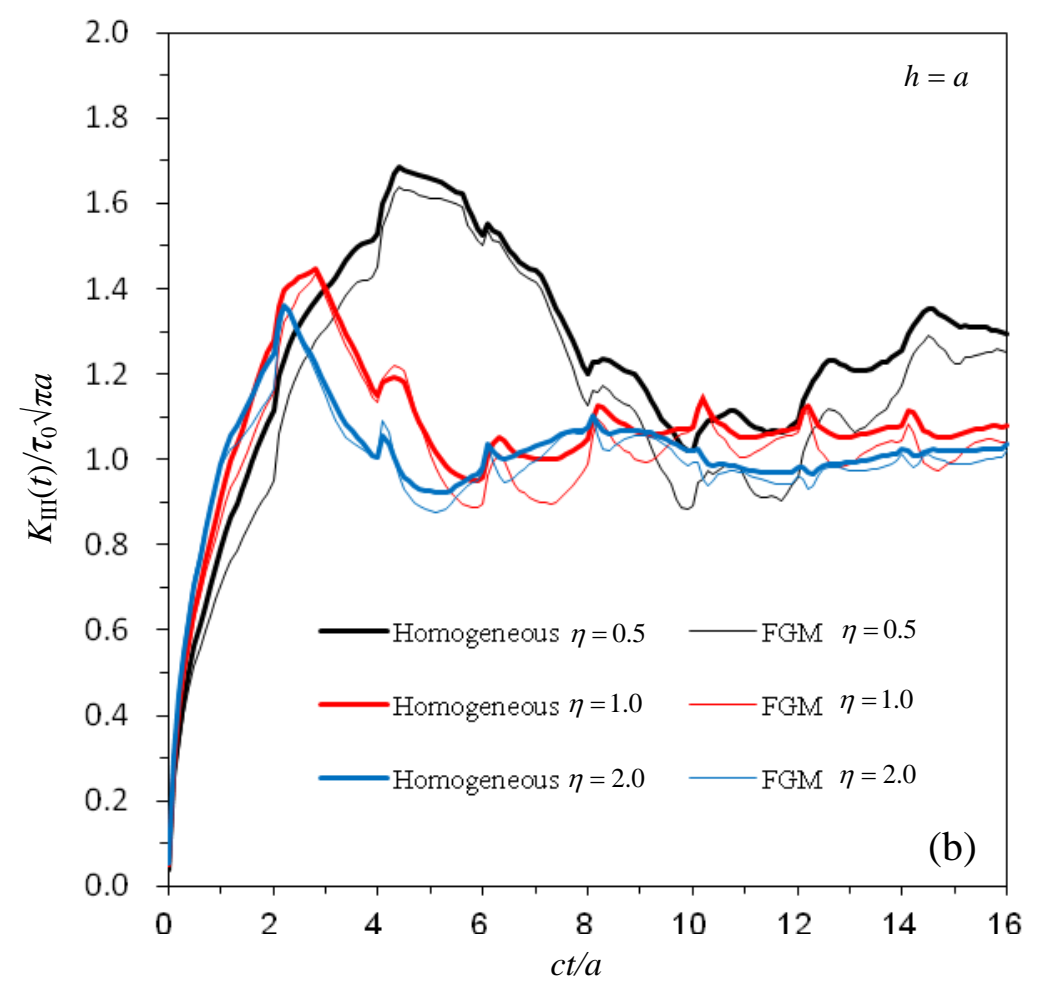

Figure 7. Normalised dynamic SIFs under Heaviside load for homogeneous and FGM coats ( $\kappa=2)$ with different orthotropic parameter ( $\eta$ ) : (a) $h=5 a$; (b) $h=a$.

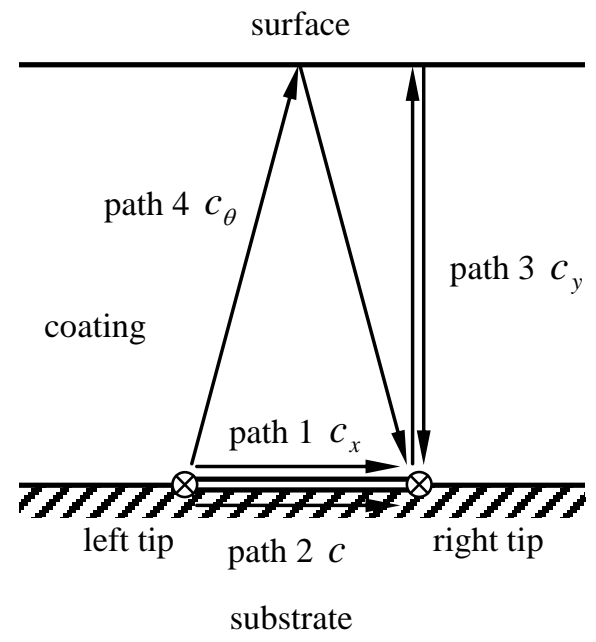

Figure 8. Different traveling paths for elastic shear waves in substrate and coating. 
Considering the right tip of crack, the dynamic SIFs are plotted in Figure 7(a) while $h=5 a$ and the paths of elasticity shear wave starting from two crack tips are shown in Figure 8. The coordinate of the first kink (A) is given by $c t_{A} / a=1$ for $\eta=2\left(c_{x}=2 c\right)$ which shows the time required for shear waves to travel from one crack tip (left) to the other tip (right) in the single layered coating (path 1 in Figure 8). The first kink is thus caused by the arrival of this shear wave in the coat although it is not very sharp. The second kink (B) is due to the shear wave in the substrate staring from crack tip (left) and traveling to the other tip (right) for all ratios of $\eta$. It is worth to notice that the time required for shear wave travelling from one tip to the other in the substrate is the same, i.e. $c t_{B} / a=2$ (path 2 in Figure 8). The coordinate of the kink (C) is $c t_{C} / a=4$ for $\eta=0.5$ which is the time required for shear wave to travel from crack tip to the other tip in the coating (path 1 in Figure 8). The kink (D) is obviously due to the arrival of the reflected shear wave from the free boundary $y=h=5 a$ starting from crack tip and the normalized time is $c t_{D} / a=10$ for both homogeneous/FGM coatings due to the speeds of shear wave are the same along $y$-axis $\left(c_{y}=c\right.$ ) (path 3 in Figure 8). However, the next kink (E) should be the arrival of the reflected shear wave from traction free boundary $(y=h=5 a)$ starting from the crack tip (left) with normalized time in total $c t_{E} / a=2 \sqrt{h^{2}+a^{2}} / a$ $=10.198$ for isotropic coating $(\eta=1$, path 4 in Figure 8). However, for orthotropic coating $(\eta=0.5)$, this wave requires normalised time about $c t_{E^{\prime}} / a=10.8$ seeing from Figure 7 (a) due to the material property travelling with speed $c_{\theta}$. In general, there are lots of kink generated by the different shear waves starting (reflecting) from crack tips (free boundary). It is not difficult to identify each sharp kink in Figure 7(b) by considering the arrival times for the elastic shear waves. In addition, in the case of $\kappa=2$, the dynamic SIFs with orthotropic FGM coatings under shear impact are slightly lower than that with homogeneous coatings.

\section{Conclusion}

Anti-plane fracture problem with interfacial crack between substrate and homogeneous /FGM coatings has been studied by using displacement discontinuity method in this paper. The fundamental solutions of displacement discontinuity on the interface are derived for both static and dynamic. Also the fundamental solution for a strip with homogeneous and FGM coats are 
included. The first kind Fredholm integral equation was numerically solved with Chebyshev series to determine stress intensity factor. It has been found that when the shear modulus of the coating is higher than that of the substrate for both two coats, the SIF is reduced slightly. The effect of orthotropic property of the coat on the stress intensity factor is also investigated. It is shown that the SIF with larger shear modulus in $x$-axis direction reduces significantly. Dynamic anti-plane fracture problems were studied in the Laplace transformed domain and the Durbin's inverse transform method was employed in order to obtain high accurate SIF in the time domain. The time dependent stress intensity factors under uniformly distributed dynamic load on the crack surfaces demonstrated the effect of elastic shear waves travelling in the materials.

\section{References}

[1] S. Suresh, A. Mortensen, Fundamentals of Functionally Graded Materials, Institute of Materials, London; 1998.

[2] Y. Miyamoto, W.A. Kaysser, B.H. Rabin, A. Kawasaki, R.G. Ford, Functionally Graded Materials: Design, Processing and Applications, Kluwer Academic Publishers, Dordrecht; 1999.

[3] D.Y. Liu, C.Y. Wang, W.Q. Chen, Free vibration of FGM plates with in-plane material inhomogeneity, Compos. Struct. 92(5) (2010) 1047-51.

[4] B.S. Aragh, M.H. Yas, Three-dimensional analysis of thermal stresses in four-parameter continuous grading fiber reinforced cylindrical panels, Int. J. Mech. Sci. 52 (2010) 10471063.

[5] S.S. Vel, Exact elasticity solution for the vibration of functionally graded anisotropic cylindrical shells, Compos. Struct. 92 (2010) 2712-2727.

[6] P. Malekzadeh, A.A. Beni, Free vibration of functionally graded arbitrary straight-sided quadrilateral plates in thermal environment, Compos. Struct. 92 (2010) 2758-67.

[7] S.M. Hosseini, F. Shahabian, Reliability of stress field in Al-Al2O3 functionally graded thick hollow cylinder subjected to sudden unloading, considering uncertain mechanical properties, Mater. Des. 31 (2010) 3748-60.

[8] Y.A. Kang, X.F. Li, Large deflections of a non-linear cantilever functionally graded beam, J. Reinf. Plast. Compos. 29 (2010) 1761-74.

[9] T.S. Cook, F. Erdogan, Stresses in bonded materials with a crack perpendicular to the 
interface, Int. J. Eng. Sci. 10 (1972) 677-697.

[10] F. Delale, F. Erdogan, On the mechanical modeling of the interface region in bonded halfplane, J. Appl. Mech. 55 (2) (1988) 317-324.

[11] F. Erdogan, The crack problem for bonded nonhomogeneous materials under antiplane shear loading, J. Appl. Mech. 52 (1985) 823-828.

[12] G.Y. Huang, Y.S. Wang, D. Gross, Fracture analysis of functionally graded coatings: antiplane deformation. Eur. J. Mech. A/Solids 21 (2002) 391-400.

[13] K.Q. Hu, Z. Zhong, B. Jin, Anti-plane shear crack in a functionally gradient piezoelectric material, Acta Mech. Solida Sinica 15 (2) (2002) 140-148.

[14] X.S. Zhang, B. Liang, A central crack of mode III in a nonhomogeneous rectangular sheet with shear modulus varying in the X-direction, Engng Fract. Mech. 34(2) (1989) 269-274.

[15] X.S. Zhang, The general solution of an edge crack off the center of a rectangular sheet for mode III, Engng Fract. Mech. 31(5) (1988) 847-855.

[16] F. Erdogan, A.C. Kaya, P.F. Joseph, The mode III crack problem in bonded materials with a nonhomogeneous interfacial zone, J. Appl. Mech. 58 (2) (1991) 419-427.

[17] Z.H. Jin,N. Noda, Crack-tip singular fields in nonhomogeneous materials, ASME J. Appl. Mech. 61(1994) 738-740.

[18] Z.H.Jin, R.C. Batra, Some basic fracture mechanics concepts in functionally graded materials, J. Mech. Phys. Solids 44 (1996) 1221-1235.

[19] Z.H. Jin, R.C. Batra, Interfacial cracking between functionally graded coatings and a substrate under antiplane shear, Int. J. Engng Sci., 34 (15) (1996) 1705-1716.

[20] H.J. Choi, Elastodynamic analysis of a crack at an arbitrary angle to the graded interfacial zone in bonded half-planes under antiplane shear impact, Mech. Res. Commun. 33 (5) (2006) 636-650.

[21] C.Y. Li, G.J. Weng, Dynamic stress intensity factors of a cylindrical interface crack with a functionally graded interlayer, Mech. Mater. 33 (6) (2001) 325-333.

[22] Y.D. Li, K.Y. Lee, D. Yao, Dynamic stress intensity factors of two collinear mode-III cracks perpendicular to and on the two sides of a bi-FGM weak-discontinuous interface, European J. Mech A/Solids 27 (2008) 808-823.

[23] R.T. Faal, A.A. Dehghan, Mode III stress intensity factors for cracked FGM rectangular plane, Engng Fracture Mech. 140 (2015) 17-30.

[24] K.Q. Hu, Z. Zhong, B. Jin, Anti-plane shear crack in a functionally gradient piezoelectric 
material, Acta Mech. Solida Sinica 15 (2) (2002) 140-148.

[25] P.H. Wen, M.H. Aliabadi, J. Sladek, V. Sladek, Displacement discontinuity method for cracked orthotropic strip: Dynamic, Wave Motion 45 (2008) 293-308.

[26] M.K. Kaya, F. Erdogan, On the solution of integral equations with strong singular kernels, Q. Appl. Math. 45 (1987) 105-122.

[27] F. Durbin, Numerical inversion of Laplace transforms: an efficient improvement to Dubner and Abate's method, Comput. J. 17 (4) (1974) 371-376. 